\title{
Clinical significance of late onset antibody-mediated rejection without donor-specific anti-human leukocyte antigen antibodies in kidney transplantation
}

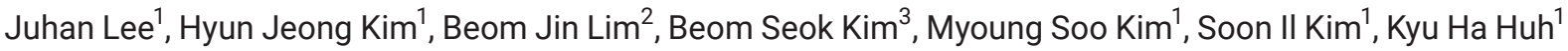 \\ ${ }^{1}$ Department of Surgery-Transplantation, Yonsei University College of Medicine, Seoul, Korea \\ ${ }^{2}$ Department of Pathology, Yonsei University College of Medicine, Seoul, Korea \\ ${ }^{3}$ Department of Internal Medicine-Nephrology, Yonsei University College of Medicine, Seoul, Korea
}

Background: Late onset antibody-mediated rejection (AMR) is a leading cause of kidney allograft failure. Its diagnosis has been based on a combination of morphologic, immunohistologic findings, and presence of donor-specific anti-human leukocyte antigen antibodies (DSA). Although the presence of DSA is no longer required for AMR diagnosis, according to Banff 2017 classification, the clinical significance of late onset AMR without DSA remains unclear. Here we compared clinical outcomes of late onset AMR with and without DSA.

Methods: We analyzed 126 cases of late AMR ( $>6$ months after transplant) that meet the Banff 2017 histologic criteria for AMR. All cases were diagnosed by for cause biopsy and grouped into DSA-positive $(n=103)$ and DSA-negative $(n=23)$ AMR groups. The estimated glomerular filtration rate (eGFR) was calculated using the Chronic Kidney Disease Epidemiology Collaboration equation.

Results: The histological picture did not differ between DSA-negative and DSA-positive AMR, with the exception of increased level of peritubular capillaritis in DSA-positive AMR. Median time from transplant to AMR diagnosis was 80 months (interquartile range, 39-118). At the time of AMR diagnosis, both groups had similar graft function $\left(36.3 \pm 16.0 \mathrm{~mL} / \mathrm{min} / 1.73 \mathrm{~m}^{2}\right.$ for DSA-negative and $39.7 \pm 20.2 \mathrm{~mL} / \mathrm{min} / 1.73 \mathrm{~m}^{2}$ for DSA-positive AMR; $P=0.408$ ). Mean eGFR after AMR were similar irrespective of the presence of DSA. There were 28 graft failures (26.2\%) in the DSA-negative and eight graft failures (27.6\%) in the DSA-positive AMR groups, which was not statistically different $(P=0.981)$.

Conclusions: In late onset AMR, there was no significant difference between AMR with and without DSA in clinical outcomes.

Corresponding author: Juhan Lee

E-mail: laplaine@yuhs.ac

(c) The Korean Society for Transplantation

This is an Open Access article distributed under the terms of the Creative Commons Attribution Non-Commercial License (http://creativecommons.org/licenses/by-nc/4.0/) which permits unrestricted non-commercial use, distribution, and reproduction in any medium, provided the original work is properly cited. 